\title{
Corrigendum
}

\section{Corrigendum to "Modeling and Measurement of Correlation between Blood and Interstitial Glucose Changes"}

\author{
Ting Shi, ${ }^{1,2,3,4}$ Dachao Li, ${ }^{1}$ Guoqing Li, ${ }^{1}$ Yiming Zhang, ${ }^{2}$ Kexin Xu, ${ }^{1}$ and $\mathrm{Luo} \mathrm{Lu}^{3,4}$ \\ ${ }^{1}$ School of Precision Instrument and Opto-Electronics Engineering, Tianjin University, Tianjin 300072, China \\ ${ }^{2}$ College of Electronic Information and Control Engineering, Beijing University of Technology, Beijing 100124, China \\ ${ }^{3}$ Department of Medicine, Division of Molecular Medicine, Harbor-UCLA Medical Center, David Geffen School of Medicine at the \\ University of California, Los Angeles, CA 90095, USA \\ ${ }^{4}$ Los Angeles Biomedical Research Institute at Harbor-UCLA Medical Center, Torrance, CA 90502, USA \\ Correspondence should be addressed to Luo Lu; lluou@ucla.edu
}

Received 19 June 2017; Accepted 11 July 2017; Published 20 August 2017

Copyright (C) 2017 Ting Shi et al. This is an open access article distributed under the Creative Commons Attribution License, which permits unrestricted use, distribution, and reproduction in any medium, provided the original work is properly cited.

In the article titled "Modeling and Measurement of Correlation between Blood and Interstitial Glucose Changes" [1], there were errors in the authors' affiliations. The correct affiliation list is shown above.

\section{Reference}

[1] T. Shi, D. Li, G. Li, Y. Zhang, K. Xu, and L. Lu, "Modeling and measurement of correlation between blood and interstitial glucose changes," Journal of Diabetes Research, vol. 2016, Article ID 4596316, 9 pages, 2016. 


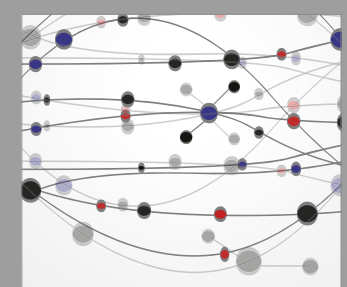

The Scientific World Journal
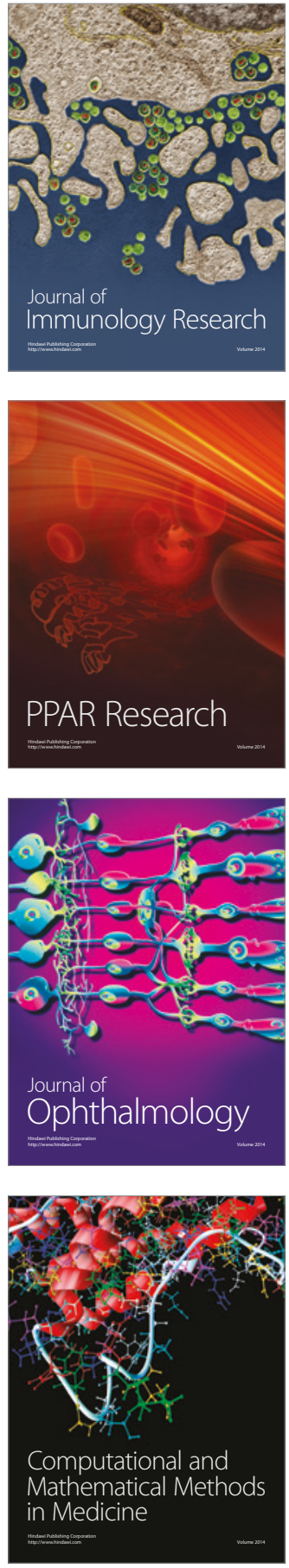

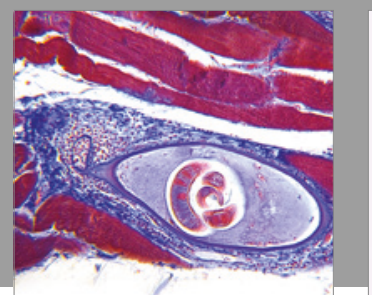

Gastroenterology Research and Practice
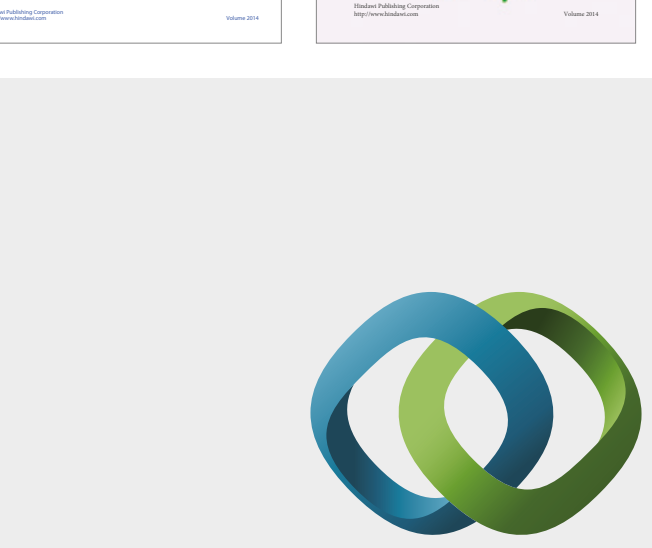

\section{Hindawi}

Submit your manuscripts at

https://www.hindawi.com
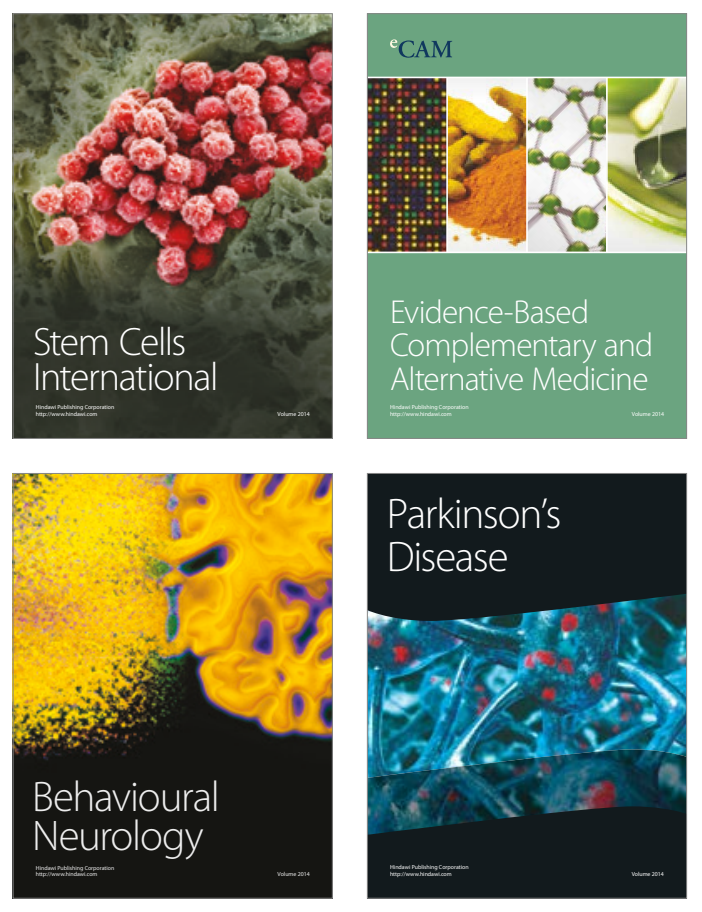
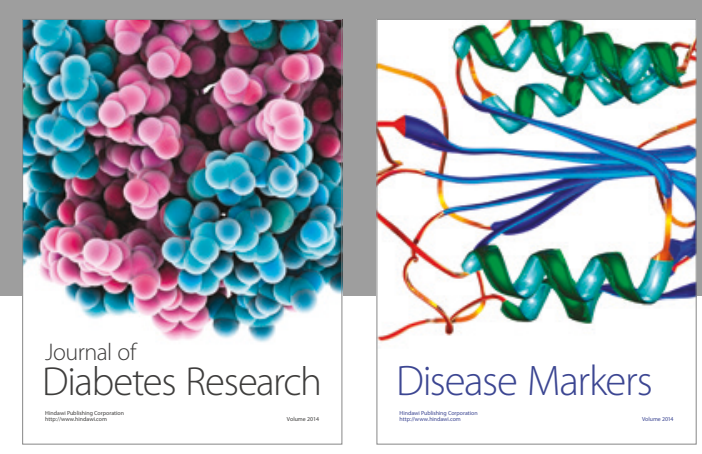

Disease Markers
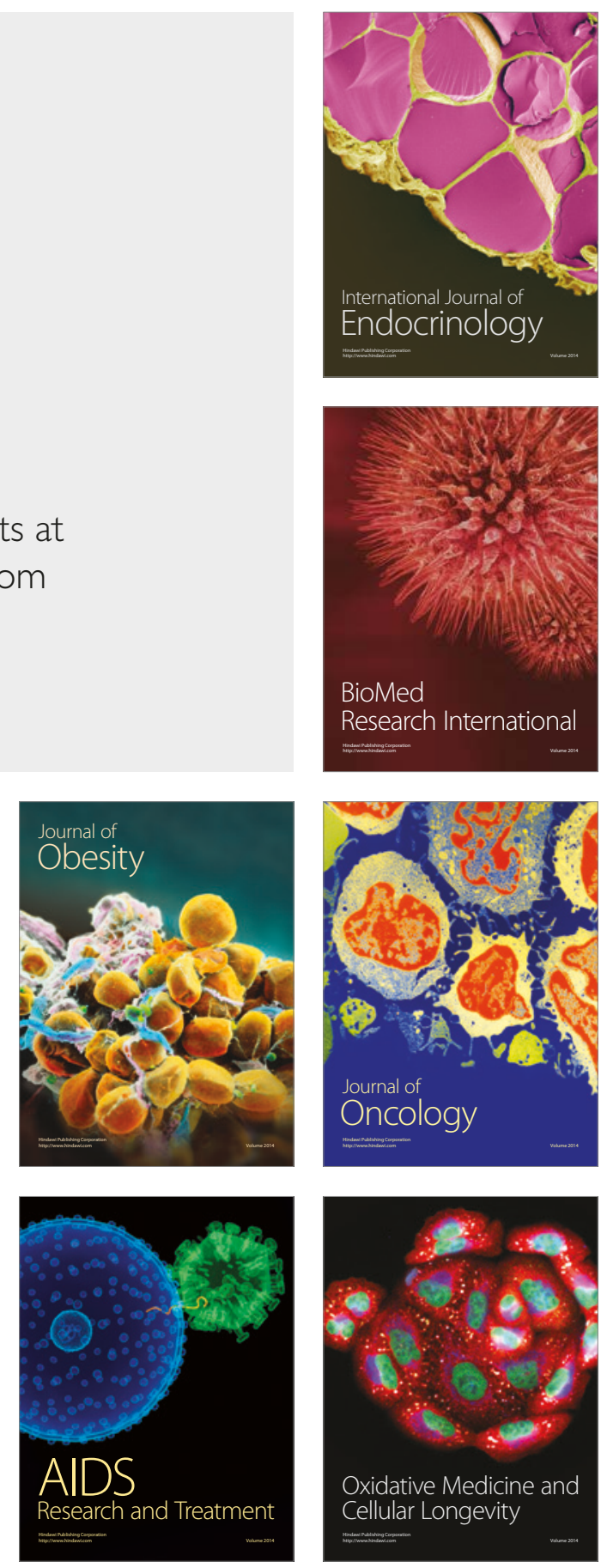